\title{
Acrosoxacin in the treatment of uncomplicated gonorrhoea
}

\author{
R J WALSH,* R SCOTT,* J B BITTINER,* M SHAHIDULlAH,† AND \\ R C B SLACK‡
}

From the *Department of Genitourinary Medicine, General Hospital, Nottingham; the +Department of Genitourinary Medicine, Lincoln County Hospital, Lincoln; and the $\$$ Department of Microbiology and PHLS Laboratory, University Hospital, Queen's Medical Centre, Nottingham

SUMMARY Acrosoxacin was given as a single $300 \mathrm{mg}$ oral dose to 105 patients with acute gonorrhoea. Of the 100 patients followed completely there was a $93 \%$ cure rate and $33 \%$ of men developed postgonococcal urethritis (PGU). This compared with a 97\% cure and 30\% PGU with 2 megaunits penicillin. The in vitro activity of acrosoxacin in Nottingham against strains of Neisseria gonorrhoeae showed that it was a highly active agent with $90 \%$ of strains inhibited by a concentration of $0.03 \mathrm{mg} / \mathrm{l}$.

\section{Introduction}

Single dose oral treatment of acute gonorrhoea is established practice in many clinics and we have had experience with minocycline in Nottingham. ${ }^{1}$ Intramuscular penicillin remains the treatment of choice in our clinic, but with an increasing prevalence of resistance to penicillin it is important that other agents should be investigated.

Acrosoxacin (Eradacin) is a new pyridyl-quinoline antibacterial agent related to nalidixic acid. It is rapidly absorbed when taken orally on an empty stomach, a dose of $300 \mathrm{mg}$ attaining a peak plasma concentration of $6.6 \mathrm{mg} / \mathrm{l}$ after two to six hours. The plasma half-life ranges from four to 11 hours and $70 \%$ of the drug is protein-bound. The drug is inactivated in the liver and its metabolites mostly excreted in the urine, although about $4 \%$ is excreted unchanged (Sterling Winthrop, unpublished data).

Warren, Shannon, and Phillips ${ }^{2}$ studied the in vitro antigonococcal activity of acrosoxacin and found that 167 of 173 isolates of Neisseria gonorrhoeae were inhibited by concentrations of $0.06 \mathrm{mg} / \mathrm{l}$ of acrosoxacin. The others were inhibited by concentrations of $0 \cdot 12-0 \cdot 25 \mathrm{mg} / 1$ of the compound. Unpublished reports of clinical trials using a single oral dose of $300 \mathrm{mg}$ of the agent have suggested that this is an effective antibacterial agent in the treatment of uncomplicated gonorrhoea.

Address for reprints: Dr R C B Slack, Department of Microbiology and PHLS Laboratory, University Hospital, Queen's Medical Centre, Nottingham NG7 2UH

Accepted for publication 30 January 1983

\section{Patients and methods}

A total of 81 men and 24 women with uncomplicated gonorrhoea attending the Nottingham or Lincoln clinics were admitted to the trial. One man with gonococcal epididymitis was also treated with acrosoxacin. Specimens for direct microscopy and culture were taken from the urethra in men and from the urethra and cervix in women. Rectal cultures were taken from 20 women who were known gonorrhoea contacts; follow up rectal cultures were taken from these patients.

Treatment was given on the basis of positive microscopy and in all cases the diagnosis was later confirmed by culture and identification of $N$ gonorrhoeae by standard methods. Patients who were known to default were excluded from the trial as were pregnant or lactating women and patients with renal or hepatic impairment.

Patients were warned of possible side effects of the drug (that is, dizziness, drowsiness, headache, and gastrointestinal upset). Avoidance of sexual activity was advised for at least two weeks in every case. The patients were asked to attend for follow up three, seven, and 14 days after treatment. On each occasion smears and cultures were repeated.

Men with symptoms or signs or both of a urethral discharge which on microscopy of a Gram smear showed $\geqslant 10$ leucocytes per microscopic field $(\times 1000)$ but no Gram-negative intracellular diplococci (GNDC), and whose urethral culture was negative, were diagnosed seven or more days after treatment as having postgonococcal urethritis (PGU) and were treated with a course of oral tetracycline. All patients 
were offered further appointments to be re-examined to exclude relapse or reinfection. Demonstration of GNDC with positive cultures within two weeks of treatment was, in the absence of admitted risk, interpreted as treatment failure. Reinfection was diagnosed when there was a history of admitted risk within that period.

Serological tests for syphilis were taken at the initial clinic attendance and repeated at the end of the period of surveillance.

Minimum inhibitory concentrations (MICs) of acrosoxacin for the isolates were determined by agar incorporation in modified New York City (MNYC) medium $^{3}$ with reference strains IRS 3 and IRS 5 as controls. $\mathbf{N}$ gonorrhoeae isolated from other patients who attended the clinics but who were not entered in the trial have been included. Strains isolated at the Public Health Laboratory, Lincoln County Hospital, were sent to Nottingham for acrosoxacin MIC testing (courtesy of the director, Dr R G Wallace.) At the same time patients with acute gonorrhoea treated with a standard regimen of 2 megaunits of procaine and benzylpenicillin (Bicillin) intramuscularly were compared with the trial group for treatment efficacy and for occurrence of PGU. The criteria for PGU were identical in both groups of patients.

\section{Results}

The total number of patients treated was 105 (81 men and 24 women). Five men defaulted, 69 were cured, and there were seven treatment failures (all male) and no reinfections. Two patients with penicillinase-producing (PPNG) strains were treated: one was cured and the other failed with acrosoxacin. The only patient with complications-epididymitis-responded well to the single ofal dose. PGU occurred at the end of the first week after treatment in $\mathbf{1 5}$ men, at the end of the second week in nine men, and at the end of the third week in one. All the 24 women treated with acrosoxacin were cured including 12 cases with rectal infection. Five patients reported transient dizziness and nausea.

In a comparative study of 300 patients with uncomplicated gonorrhoea who were treated with penicillin, $97 \%$ were cured and $30 \%$ of the men developed PGU.

The in vitro sensitivities of gonococci isolated during the trial period are shown in figs 1 and 2 and the table. No strains were found to be completely resistant to acrosoxacin. The strain isolated with an MIC of $0.125 \mathrm{mg} / \mathrm{l}$ was from a treatment failure. This strain was penicillin resistant $(\mathrm{MIC}=1 \mathrm{mg} / \mathrm{l})$ but non- $\beta$-lactamase-producing. One $\beta$-lactamase producing strain from Lincoln had an MIC of acrosoxacin of $0.015 \mathrm{mg} / \mathrm{l}$ and the patient was cured.

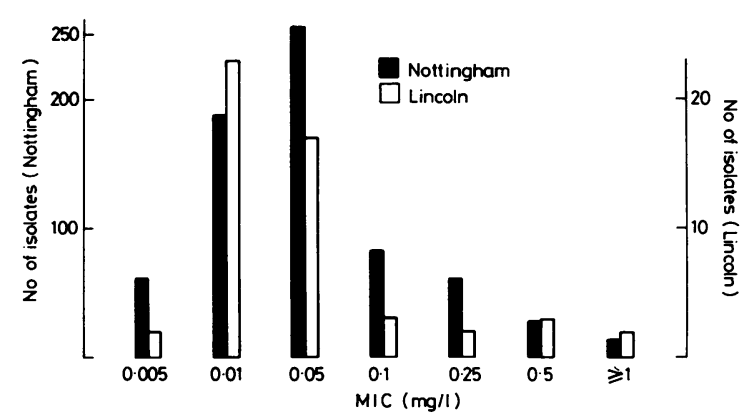

FIG 1 In vitro sensitivities to penicillin of $N$ gonorrhoeae isolated in Lincoln and Nottingham.

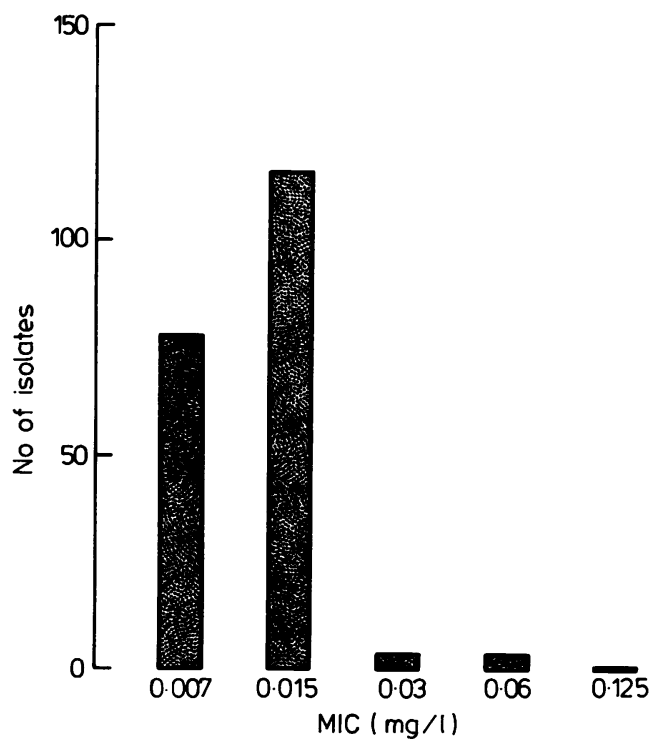

FIG 2 In vitro sensitivities to acrosoxacin of $N$ gonorrhoeae isolated in Nottingham.

TABLE Antimicrobial susceptibilities of isolates of $N$ gonorrhoeae to penicillin and acrosoxacin

\begin{tabular}{llll}
\hline & \multicolumn{2}{l}{ Penicillin } & \\
\cline { 2 - 3 } MIC & Nottingham & Lincoln & Acrosoxacin \\
\hline & & & \\
Mode & 0.05 & 0.01 & 0.015 \\
$90 \%$ & 0.5 & 0.25 & 0.03 \\
$50 \%$ & 0.1 & 0.05 & 0.03 \\
Mean & 0.09 & 0.07 & 0.014 \\
\hline
\end{tabular}

MIC = minimum inhibitory concentration 
The other PPNG strain from Nottingham had an MIC of acrosoxacin of $0.06 \mathrm{mg} / \mathrm{l}$ although treatment with acrosoxacin was unsuccessful. No increase in the MIC of acrosoxacin was detected in the isolates from the treatment failures.

\section{Discussion}

A single $300 \mathrm{mg}$ dose of acrosoxacin in patients in our clinics gave a cure rate comparable with that of the standard penicillin regimen. Acrosoxacin was acceptable to all patients as there was a low incidence of side effects. Although there was an increase in the number of patients with PGU compared with that resulting from penicillin treatment the difference was not significant.

Most isolates of $N$ gonorrhoeae in this region are sensitive to penicillin which remains the treatment of first choice. The increasing prevalence of resistant strains reported nationally ${ }^{4}$ and noticed locally may, however, cast doubt on this approach in which case acrosoxacin would be a useful drug for the treatment of acute uncomplicated gonorrhoea.
We thank the staff of the Nottingham and Lincoln clinics and PHLS laboratories; Sterling Winthrop o Ltd for financial support; Dr D S Segal, medical director, for his active assistance; and Mrs A Devlin for her secretarial help.

A summary of this paper was presented to the annual spring meeting of the MSSVD, St Helier, Jersey, 1982.

\section{References}

1. Shahidullah M. Single-dose treatment of uncomplicated $\vec{\omega}$ gonorrhoea in males with minocycline. $\mathrm{Br} J$ Vener Dis 1975;51:97-8.

2. Warren CA, Shannon KP, Phillips I. In vitro antigonococcal activity of rosoxacin (WIN 35213). Br J Vener Dis 1981;57:33-5.

3. Young $\mathrm{H}$. Cultural diagnosis of gonorrhoea with modified New York City (MNYC) medium. Br J Vener Dis 1978;54:36-40.

4. McCutcheon JA, Adler MW, Bernie JRH. Penicillinaseproducing Neisseria gonorrhoeae in Great Britain 1977-81: alarming increase in incidence and recent development of endemic transmission. Br Med J 1982; 285:337-40. 